\title{
Buchbesitz und geistiger Horizont. Zur Rekonstruktion frühneuzeitlicher Privatbibliotheken
}

Wie sah es im Körper, im Geist und in der Seele unserer Vorfahren aus? Welchen Veränderungen war ihr körperlicher, geistiger und seelischer $\mathrm{Zu}$ stand unterworfen? Historiker, die das Werden komplexer menschlicher Gesellschaften untersuchen, müssen sich solche Fragen stellen ${ }^{1}$. Was folgt, sind einige Antworten eines Frühneuzeitlers betreffend den „geistigen Horizont” von Menschen, die im späten 17. und im 18. Jahrhundert auf dem Gebiet der schweizerischen Eidgenossenschaft, des Corpus helveticum, lebten ${ }^{2}$. Der vorsichtige Begriff „geistiger Horizont” - oder das englische mindscape - steht für die Schwierigkeit, in den Geist der Menschen einzudringen, für die Einsicht, dass wir meist bloss seine Ausdehnung, seine Umrisse und seine ungefähre Struktur skizzieren können.

$\mathrm{Zu}$ Menschen, die wir nicht mehr befragen können, haben wir keinen unmittelbaren Zugang. Im Fall des Zugangs zu ihrem Geist, drängt sich für die Frühe Neuzeit als Vermittler das (gedruckte) Buch auf: das besessene, gelesene, verstandene oder in Taten umgesetzte Buch. Wobei zu bedenken ist, dass Bücher nicht die einzige oder gar die wichtigste Quelle geistiger Nahrung jener Zeit waren und dass nicht nur in Büchern gelesen wurde. Welche Art von Informationsquellen stehen dem Historiker zur Verfügung, der sich einerseits für die Gesellschaft als Ganzes interessiert, anstatt lediglich einzelne ihrer Geistesgrössen herauszupicken, und der anderseits längerfristige Entwicklungen ins Auge fasst, anstatt sich mit der punktuellen Beobachtung eines bestimmten Zeitabschnitts zufrieden zu geben? Es sind, soweit ich sehe, vor allem deren zwei: Nachlassinventare und Versteigerungsinventare (in Folge des Ablebens, eines Konkurses oder einer Konfiskation). Zur Veranschaulichung seien hier einige Archivbestände aufgezählt, die mir bekannt sind und die ich ganz oder teilweise ausgewertet habe.

1 Siehe dazu N. Furrer, Geschichtsmethode: Eine Einführung für Humanhistoriker, 2., überarbeitete und erweiterte Ausgabe, Zürich 2014, S. 27-28.

2 Zur Ausdehnung des Territoriums und Zusammensetzung des Corpus helveticum, siehe beispielsweise Historischer Atlas der Schweiz - Atlas historique de la Suisse-Atlante storico della Svizzera, hg. von H. Ammann und K. Schib, 2. Auflage, Aarau 1958, S. 33. 
Im Staatsarchiv des Kantons Bern:

- B IX 1408-1409: Geltstagsrödel für Geltstage im Bezirk der Stadt Bern allein, 1646-1666;

- B IX 1410-1439: Geltstagsrödel für Geltstage im Bezirk der Stadt Bern und des Stadtgerichts Bern, 1666-1765;

- B IX 1440-1461: Geltstagsrödel (mit Verzeichnis der Geltstager auf dem Rücken der Bände), 1714-1797;

- B IX 1463-1548: Geltstagsrödel (in Einzelstücken, in alphabetischer Folge der Geltstager), 2. Hälfte des 17. Jhs. bis 1831;

- Bez Aarwangen A 345-371: Geltstagsrödel des ehemaligen Amts Aarwangen, Bd. 1-26, 1678-1798;

- Bez Wangen A 460-507: Geltstagsrödel des Amts Wangen, Serie 1, Bd. [1-10], 1701-1798.

Im Staatsarchiv des Kantons Solothurn:

- Ganten und Steigerungen in der Stadt Solothurn, Bd. [1-23], 1671-1803.

In den Archives de l'ancien Evêché de Bâle (Porrentruy):

- Actes de l'ordre judiciaire déposés dans les Archives de la ci-devant Principauté de Bâle: Porrentruy, ville, inventaires et partages, $\mathrm{N}^{\text {os }} 10-35$ ( $1^{\text {ère }}-26^{\text {e }}$ divisions), 1618-1792;

-Actes de l'ordre judiciaire: Porrentruy, ville, discussions et montes, $N^{\text {os }}$ 36-48 ( $1^{\text {ère- }} 11^{\text {e }}$ divisions), 1595-1792;

-Actes de l'ordre judiciaire: Delémont, ville, inventaires et partages, Nos 3-7 (1 $1^{\text {ère }} 5^{\mathrm{e}}$ divisions), 1655-1792.

In den Archives de la Ville de Lausanne:

- Chavannes D 533-539: Inventaires de biens faits sur ordre du Conseil, $1694-1798^{5}$.

In den Archives cantonales vaudoises (Chavannes-près-Renens):

- Bih 65/1-17: Liasse d'inventaires de biens [provenant de la Vallée de Joux], 1788-1794;

3 Siehe N. Furrer, Schriftkunde und Textedition: Anleitung zum Umgang mit frühneuzeitlichen Manuskripten am Beispiel Berns, Zürich 2016, S. 75-78: Die Bibliothek des Notars und Wirts Christoffel Kienberger im Jahre 1664.

4 Siehe N. Furrer, Die Bibliothek eines Stadtberner Handwerkers des 17. Jahrhunderts, in: Berns mächtige Zeit: Das 16. und 17. Jahrhundert neu entdeckt, hrsg. von A. Holenstein [u. an.], Bern 2006, S. 362; N. Furrer, Bücher machen Leute: Berner Privatbibliotheken des 18. Jahrhunderts in gesellschaftlicher Perspektive, in: Berns goldene Zeit : Das 18. Jahrhundert neu entdeckt, hg. von A. Holenstein [u. an.], Bern 2008, S. 229-231; N. Furrer, Des Burgers Buch: Stadtberner Privatbibliotheken im 18. Jahrhundert, Zürich 2012, 824 S.

5 N. Furrer, La bibliothèque du conseiller lausannois Benjamin Milot en 1757, „,Revue historique vaudoise" 2012, Vol. 120 , S. 297-314. 
- Bit 58-137, 217, 246, 252, 455-488: Cours de justice diverses: District d'Yverdon, 1757-1797.

In den Archives de l'Etat de Neuchâtel:

- Archives judiciaires: La Côte, $\mathrm{N}^{\circ} 74$ : Montes, 1748-1822;

- Archives judiciaires: La Chaux-de-Fonds, $N^{\circ}$ 243: Inventaires de discussion - inventaires de décrets de biens, 1781-1789;

- Archives judiciaires: [Ville de] Neuchâtel, $\mathrm{N}^{\circ}$ 237: Montes (enchères), 1792-1811.

Die Liste zeigt, dass an historischem Quellenmaterial wahrlich kein Mangel herrscht, zumal sie mühelos durch Bestände aus den erwähnten und aus anderen - nicht nur schweizerischen - Archiven verlängert werden könnte.

Vermerkenswert ist ausserdem: Die erwähnten Inventare stammen aus reformierten und katholischen Gebieten ${ }^{6}$, aus deutsch- und französischsprachigen Gegenden sowie aus städtischen und ländlichen Ortschaften. Von der gesellschaftlichen Spannbreite, die sie abdecken, zeugt die Vielfalt der Berufe, denen die Vorsteher der inventarisierten Haushalte nachgehen. In der Stadt Bern beispielsweise sind es für die zweite Hälfte des 17. Jahrhunderts folgende Ämter und Berufe: Ratsherr (Mitglied des Kleinen Rates), Landvogt, Grossrat, Kastlan, Spitalmeister, Advokat, Pfarrer, Kantor, Schreiber (Sekretär), Offizier, Rittmeister, Wachtmeister, Förster, Apotheker, Wirt, Weinhändler, Spezereihändler, Schlosser, Tischmacher, Schneider, Säckler (Sackmacher), Färber, Flachmaler, Schärer, Hutstaffierer (Hutschmücker) Schuhmacher, Metzger, Bäcker, Überreiter, Stadtläufer, Totengräber ${ }^{7}$. Stark untervertreten - mit ein paar wenigen Prozenten - sind hingegen die Frauen; ganz abwesend die Kinder und Jugendlichen ${ }^{8}$.

Nachlassinventare und Versteigerungsinventare sind für die gegebene Forschungsperspektive insofern relevant, als unter den Besitztümern, die sie auflisten, Buchtitel vorkommen. In Wirklichkeit ist dies bei weitem nicht immer der Fall, wie es die Auswertung von vier Quellenkorpora belegt: eines aus der Stadt Bern in der zweiten Hälfte des 17. Jahrhunderts und drei aus den Städten Bern und Lausanne im 18. Jahrhundert ${ }^{9}$. Schenkt man den

\footnotetext{
${ }^{6}$ Zu letzteren gehören Solothurn und das Fürstbistum Basel (Ancien Evêché de Bâle). - Zur damaligen Verteilung der Konfessionen in der Schweiz, siehe Historischer Atlas der Schweiz..., S. 35.

7 Die Liste bezieht sich auf die Haushaltsvorsteher im Besitz von Büchern. - Für das 18. Jahrhundert, siehe N. Furrer, Des Burgers Buch..., S. 5-6, 73-106.

8 Siehe N. Furrer, Des Burgers Buch..., S. 23.

9 Die Quellenkorpora sind: Im Staatsarchiv Bern: B IX 1408-1415: Geltstagsrödel für Geltstage im Bezirk der Stadt Bern allein, Bd. 1-8, 1646-1699; B IX 1416-1426: Geltstagsrödel für Geltstage im Bezirk der Stadt Bern und des Stadtgerichts Bern, 1700-1750; B IX 1539-1548: Geltstagsrödel (in Einzelstücken, in alphabetischer Folge der Geltstager), Buchstaben W-Z (Eckdaten: 1700-1803); Chavannes D 535-539: Inventaires de biens faits sur ordre du Conseil, 1751-1798.
} 
Quellen Vertrauen, besassen zwei Drittel bis drei Viertel der Haushalte kein einziges Buch. Zudem besassen wiederum die Hälfte bis drei Viertel der mit einer Bibliothek bestückten Haushalte höchstens zehn Werke, das heisst in unserer Terminologie - „Kleinstbibliotheken” ${ }^{10}$. Am aussagekräftigsten, den geistigen Horizont der Buchbesitzer betreffend, scheinen im übrigen die „kleinen Bibliotheken” (mit 11 bis 50 Titeln) und die „mittleren Bibliotheken” (mit 51 bis 300 Titeln), weniger hingegen die „Grossbibliotheken” (mit über 300 Titeln), besonders die viele Hundert oder Tausende von Werken zählenden Bestände (siehe Tabelle 1) ${ }^{11}$.

Was Nachlassinventare und Versteigerungsinventare an Informationen liefern, sind mehr oder weniger detaillierte und mehr oder weniger verlässliche Informationen zu Druckschriften (Büchern, Broschüren, Zeitschriften) oder - seltener - ,geschriebenen”, das heisst handschriftlichen Büchern. Ein paar Beispiele aus den Versteigerungsinventaren (Geltstagsrödeln) der Stadt Bern, mit dem Jahr der Niederschrift des betroffenen Inventars:

- Johann Peters Zwenglen Formular buch (1661);

- Gärteners Anbind Briefflein in 8 (1674);

- Eine Außlegung über die Psalmen Davids in folio (1689);

- Joseph Alleen Christliche Send Brieffen (1694);

- Hooffschul (1720);

- H. Wagners Volckbesatzung (1720);

- Eine alte geschriebene Statt Satzung (1726);

- Von der Alten Europeischen fama 12 Bänd in 8vo. à 10 bz der Band (1737);

-Würtenbergischer Stammbaum. Stuggard 1734. in fol. (1737);

- Passepartout de l'Eglise Romaine (1772);

- Un petit Livre de l'éducation des enfants, traduit de l'anglois, Amsterdam 1695 en parchemin (1776);

- Gülers Rhetia fol. (1798).

Informationen dieser Art liefern dem Forscher lediglich das Rohmaterial, das er erst einmal bearbeiten und in ein Objekt verwandeln muss, bevor er dieses dann einer wissenschaftlichen Analyse unterziehen kann. Mit anderen

\footnotetext{
10 Siehe N. Furrer, Des Burgers Buch..., S. 492-495, Tab. 25 und 26. - Die noch nicht veröffentlichten Zahlen für die Stadt Bern in der zweiten Hälfte des 17. Jahrhunderts lauten: Inventare bzw. Haushalte: 172 (100\%); Haushalte ohne Bücher: 109 (63\%); Haushalte mit Kleinstbibliotheken: 31 (49\%) von 63 Haushalten mit Büchern. Die Zahlen für Lausanne in der zweiten Hälfte des 18. Jahrhunderts lauten: Gesamtzahl der Inventare bzw. Haushalte: 360 (100\%); Haushalte ohne Bücher: 226 (63\%); Haushalte mit Kleinstbibliotheken: 105 (78\%) von 134 Haushalten mit Büchern.

11 Zur vorgeschlagenen Hierarchie der frühneuzeitlichen Privatbibliotheken, siehe N. Furrer, Des Burgers Buch..., S. 25-26, 50, Tab. 10.
} 
Worten, es gilt, anhand der Buchtitel-Liste in einem Inventar - auf dem Papier - die Privatbibliothek der Person zu rekonstruieren, dessen Güter vom Inventar erfasst wurden. Es handelt sich dabei, wohl bemerkt, um den Bestand der Bibliothek zu einem bestimmten Zeitpunkt; Nachlassinventare und Versteigerungsinventare erlauben es kaum, die Entwicklung einer Bibliothek im Laufe der Jahre ihrer Existenz zu verfolgen.

Bibliotheksrekonstruktionen sind den Familienrekonstruktionen der Bevölkerungshistoriker vergleichbar. Man konstruiert ein historisches Forschungsobjekt, indem man etwas rekonstruiert - Bibliotheken bzw. Familien - das einstmals existierte und dann verschwunden ist ${ }^{12}$.

\section{Bibliotheksrekonstruktion ${ }^{13}$}

Wie rekonstruiert man eine Privatbibliothek anhand eines Nachlass- oder Versteigerungsinventars?

In einem ersten Schritt muss man entscheiden, welche Informationen zu jeder Bibliothek dem Inventar entnommen werden sollen, durch welche weiteren Informationen diese, wenn möglich, zu ergänzen sind und auf welche Weise die zusammengetragenen Daten angeordnet werden. Die Antwort auf diese Fragen ist folgende:

Die den Bibliotheksbesitzer betreffenden biografischen Daten, die aus dem Inventar und anderen Quellen stammen, stehen in einer Anmerkung zur Titelzeile der Bibliothek. Zum Beispiel: Bibliothek des Berner Notars Johann Rudolf Gaudard (1662-1719) anhand seines Geltstagsrodels vom 4. Juli 1719. Anmerkung: ,Johann Rodolff” Gaudard wurde am 15. Juni 1662 als Sohn von Samuel Gaudar[d] und Elisabeth Herman[n] in Bern getauft (siehe BBB, Burger Taufrode $1^{14}$ IX, S. 165, Nr. 6). Am 27. April 1683 wurde „H[ans] Rudolff Gaudard” als Notar vereidigt (siehe Staatsarchiv des Kantons Bern - StaBE, A I 783: Matrikelbücher der Notare, Bd. 3, S. 220). Er heiratete am 22. August 1684 in Bolligen Ursula Ernst (siehe BBB, Burger Eherodel V, S. 219). Ursula Ernst wurde am 19. Juli 1665 als Tochter von Johann Heinrich Ernst und Ursula Thormann in Bern getauft (siehe BBB, Burger Taufrodel IX, S. 205 Nr. 3) $)^{15}$.

12 Historiker rekonstruieren zum Beispiel auch: Währungen (Systeme von Geldwährungseinheiten), Patenschaften (Beziehungsgeflechte zwischen Paten und ihren Patenkindern sowie deren Eltern), Netze von Briefkorrespondenzen, Mitgliedschaften von Sozietäten, Regierungsund Verwaltungsstrukturen bzw. -organigramme.

13 Vgl. N. Furrer, Des Burgers Buch..., S. 25-26.

14 Burgerbibliothek Bern (BBB), VA BK: Burger(liche) Taufrodel, 1530-1896, Bern. Seit 2009 digitalisiert

15 Vgl. N. Furrer, Des Burgers Buch..., S. 161. 
Die Daten zur Bibliothek sind horizontal auf vier Spalten verteilt, nämlich:

(1) die Nummer des Werkes (von 1 bis n) aufgrund der Reihenfolge der Titel in der rekonstruierten Bibliothek;

(2) die Transkription der Titel- bzw. Werkangabe, wie sie in der Quelle steht;

(3) die detaillierte bibliografische Beschreibung des Werkes, das - ohne Zweifel, wahrscheinlich oder möglicherweise - den Angaben in der Quelle entspricht;

(4) der von den Verfassern des Inventars geschätzte Wert des Werkes (in Geldwährungseinheiten), falls er erwähnt wird.

Die vertikale Gliederung der Bibliotheken ist dreistufig. In den meisten Inventaren stehen die Titel nicht oder nur zum Teil in einer sinnvollen Reihenfolge. Oft sind sie einfach nach Formaten - in-2, in-4, in-8 usw. - angeordnet (siehe Tabelle 2).

In der Rekonstruktion wird der Bibliotheksbestand aufgrund der verschiedenen sozialen Funktionen, welche die Lektüre eines Buches erfüllen kann, auf fünf Gruppen verteilt: Orientierungs-, Arbeits-, Bildungs-, Unterhaltungs- und Erbauungslektüre. Betrachtet man die Lektüre als eine Tätigkeit, die in verschiedenen Lebensbereichen ausgeübt wird und verschiedene soziale Funktionen haben kann, so lassen sich in der Tat die erwähnten fünf Arten von Lektüre unterscheiden. Orientierungs- oder informative Lektüre betreibt man beim Verwalten seines Lebens als Mitglied der Gesellschaft; sie dient der Vergesellschaftung des Individuums. Arbeits- oder professionelle Lektüre ist an die Berufsarbeit gebunden und dient als solche der Lebenserhaltung und Einkommenssicherung. Bildungs- oder ,szientifische” Lektüre pflegt man beim (ausserberuflichen) Lernen zum Zweck der Wissensvermehrung. Unterhaltungs- oder belletristische Lektüre ist spielerischer Natur und verschafft Lustgewinn und Entspannung. Erbauungs- oder religiöse Lektüre schliesslich ist ein Glaubensakt, sichert den Gnadenerwerb und spendet Trost (siehe Tabelle 3). Über die Reihenfolge der Lektürearten bei der Rekonstruktion kann gestritten werden. So könnte man beispielsweise die Erbauungslektüre an die erste statt an die letzte Stelle setzen.

Innerhalb der fünf Gruppen werden die Werke, wenn sie zahlreich sind, nach Wissensbereichen (bei der Arbeits- und Bildungslektüre) bzw. nach ihrer Thematik oder Gattung geordnet. Auf der untersten Gliederungsstufe stehen die Werke in der alphabetischen Reihenfolge ihrer Verfasser bzw. Titel (bei anonymen oder kollektiven Werken).

In einem zweiten Schritt muss man festlegen, wie die einzelnen Werke bzw. Bücher formal beschrieben werden. Das Beschreibungsprotokoll umfasst folgende Elemente: Vorname(n) und Namen des Verfassers, Titel, 
Untertitel und „Titelblattkommentare”, Verlagsort(e), Verleger ${ }^{16}$, Erscheinungsdatum, Seitenzahlen (bei einbändigen Werken) oder Anzahl Teile bzw. Bände, Format. Die Beschreibungen werden ergänzt durch Angaben im Anmerkungsapparat zur Erstausgabe, zu weiteren Ausgaben, zur Ausgabe in der Ursprungssprache (bei Übersetzungen), durch Hinweise auf biografische Informationen über die Verfasser (siehe Tabelle 4 und Abbildung 1).

Auf eine systematische Beschreibung der Werke in materieller Hinsicht kann verzichtet werden. Ob ein Buch gebunden ist oder nicht, illustriert oder nicht, vollständig oder nicht, in gutem Zustand oder nicht, erfahren wir nur dann, wenn das Inventar dies eigens vermerkt ${ }^{17}$.

Zum Gesagten seien zwei Bemerkungen angefügt. Erstens, die Gegenüberstellung der - oft ungenauen - Buchbeschreibung, wie sie die Quelle bietet, mit der vom Historiker vorgeschlagenen Beschreibung ermöglicht es dem Leser, die von diesem getroffene Wahl kritisch zu hinterfragen. Zweitens, eine Bibliotheksrekonstruktion, die diesen Namen verdient, darf sich den Aufwand der hier propagierten detaillierten Beschreibung der Werke nicht ersparen. Es ist dies die unerlässliche Bedingung einer vertieften Analyse.

\section{Analyseraster ${ }^{18}$}

An die rekonstruierte Bibliothek legt man ein Analyseraster an, das dazu dient, die Bibliothek danach abzufragen, ob sie überhaupt etwas bzw. was sie über den geistigen Horizont ihres Besitzers aussagt.

Es lässt sich ein verfeinertes Analyseraster denken, das sechs Dimensionen umfasst: eine räumliche, zeitliche, soziale, politische, kulturelle und historische (siehe Tabelle 5). Mit räumlicher und zeitlicher Dimension ist die geografische Herkunft der Werke (Verlagsorte der Bücher) bzw. ihr Alter (Entstehungs- oder Erscheinungsdatum der Texte) gemeint. Die soziale Dimension verweist auf die Schicht-, Geschlechts-, Alters- und Berufsspezifik der Werke, das heisst auf ihre Angemessenheit an oder Ausrichtung auf einen höheren oder niedereren Stand bzw. Rang, auf Männer oder Frauen, auf Erwachsene, Kinder oder junge Menschen, auf diese oder jene Berufsgruppe. Unter politischer Dimension ist den Grad der Bedrohlichkeit oder ,Zensurwürdigkeit” der Werke zu verstehen, das Mass der Kritik ihrer Verfasser an den staatlichen oder kirchlichen Obrigkeiten und den öffentlichen Moralvorstellungen. Die kulturelle Dimension bilden: die Sprache(n), die in den Werken verwendet werden; der Denkbereich (Glauben, Ethik, Noetik, Ästhetik), dem die Werke zuzuschreiben sind; der Wissens-, Fachoder noetische Bereich (Theologie, Recht, Medizin, Philosophie, Geschichte,

\footnotetext{
16 Auf die Angabe des Verlags bzw. (kommerziellen) Verlegers kann verzichtet werden.

17 Siehe N. Furrer, Des Burgers Buch..., S. 49, Tab. 9.

18 Vgl. N. Furrer, Des Burgers Buch..., S. 30-31.
} 
Geografie, Naturwissenschaften), zu dem die Werke gezählt werden; die Textgattung, der die Werke angehören; die Konfession, Religion oder Weltanschauung der Verfasser der Werke. Die historische Dimension schliesslich besteht in der Zukunftsträchtigkeit der Werke, ihrem Überdauern in späteren Zeiten, ihrem Aktualisierungspotential, ihrer „Nachhaltigkeit”.

In vielen Fällen ist dieses detaillierte Analyseraster nicht anwendbar, da die nötigen Informationen fehlen oder die Beantwortung der Fragen zuviel Zeit kosten würde ${ }^{19}$. Ein vereinfachtes und schematisierendes Analyseraster beschränkt sich auf vier Parameter, deren Quantifizierung in der Regel recht einfach ist:

(1) Thematik: das zahlenmässige Verhältnis zwischen weltlichen und geistlichen, profanen oder religiösen Werken;

(2) Funktion: das Verhältnis zwischen extraprofessionellen und berufsbezogenen Werken, zwischen Nichtarbeits- und Arbeitslektüre;

(3) Alter: das Verhältnis zwischen neuen und alten Werken;

(4) Sprache: das Verhältnis zwischen fremdsprachigen und muttersprachlichen Werken.

Die Resultate der Analyse lassen sich auf einem Viereck darstellen, dessen vier Seiten je einen Parameter symbolisieren und, ausgehend von der Mitte, je zwei Prozentskalen tragen. Die errechneten Daten (Prozentzahlen) werden auf jeder Seite eingetragen und geradlinig miteinander verbunden ${ }^{20}$.

Das dadurch entstehende Viereck visualisiert durch seine Lage, Form und Ausdehnung den geistigen Horizont des Bibliotheksbesitzers (siehe Abbildung 2$)^{21}$.

\section{Forschungstechniken}

Wissenschaftliches Forschen besteht nicht nur aus Methode und Strategien, sondern auch aus Techniken. Es erfordert den Umgang mit Arbeitsinstrumenten; es braucht handwerkliches Geschick, die Handhabung von - bereits vorhandenen oder selber hergestellten - Werkzeugen ${ }^{22}$.

Womit muss umzugehen wissen, wer frühneuzeitliche Privatbibliotheken (aus dem deutschen Sprachbereich) rekonstruieren will? Unter anderem:

- mit den einschlägigen Archivinventaren;

- mit Manuskripten in deutscher Kurrentschrift;

- mit den Regeln der Transkription bzw. Transliteration der Texte in aktuelle lateinische Druckschrift;

19 Siehe einen ansatzweisen Versuch in N. Furrer, Des Burgers Buch..., S. 517-530.

20 Siehe N. Furrer, Des Burgers Buch..., S. 70, Tabelle 24: Der ,geistige Horizont" des frühneuzeitlichen Lesers: Schematisierung; sowie S. 497-516.

${ }^{21}$ Siehe auch N. Furrer, Geschichtsmethode..., S. 64-65.

22 Siehe dazu N. Furrer, Geschichtsmethode..., S. 12-15. 
- mit Bibliothekskatalogen und -metakatalogen, insbesondere mit dem Karlsruher virtuellen Katalog;

- mit den (internationalen) Regeln der Buchbeschreibung;

- mit biografischen Wörterbüchern wie der Biographie universelle ancienne et moderne, der Allgemeinen Deutschen Biographie, der Deutschen Biographischen Enzyklopädie, dem Oxford Dictionary of National Biography, dem Dizionario biografico degli Italiani, dem Diccionario biográfico español usw., zur Identifizierung der Autoren, Übersetzer und Herausgeber der verzeichneten Werke;

- mit bibliografischen Nachschlagewerken wie beispielsweise Gerhard Dünnhaupts Personalbibliographien zu den Drucken des Barock;

- mit einschlägigen Quellen (insbesondere Pfarrbüchern), Hilfsmitteln und Studien zu den Biografien der Bibliotheksbesitzer ${ }^{23}$.

Tabelle 1: Hierarchie der Privatbibliotheken des 18. Jahrhunderts ${ }^{24}$

\begin{tabular}{|c|c|c|c|}
\hline $\begin{array}{l}\text { Umfang } \\
\text { (Anzahl } \\
\text { Werke) }\end{array}$ & $\begin{array}{l}\text { Gestalt } \\
\text { (Funktionen) }\end{array}$ & $\begin{array}{l}\text { Besitzer } \\
\text { (Bildungsstand) }\end{array}$ & $\begin{array}{l}\text { Bezeichnung } \\
\text { (Grössenordnung) }\end{array}$ \\
\hline $1-10$ & unifunktiona & Geschulte & Kleinstbibliothek \\
\hline $11-50$ & plurifunktional & Gebildete & Kleinbibliothek \\
\hline $51-300$ & multifunktional & Kultivierte & mittlere Bibliothek \\
\hline $301 \rightarrow$ & multifunktional & Gelehrte & Grossbibliothek \\
\hline
\end{tabular}

Tabelle 2: Darstellungsweise der Bibliotheken ${ }^{25}$

A. Horizontale Anordnung der Informationen (in vier Spalten)

1. Stelle des Werks in der rekonstruierten Bibliothek: Nummerierung

2. Erwähnung des Werks in der Quelle: Transkription

3. Bibliographische Beschreibung des mit dem in der Quelle erwähnten (möglicherweise) identischen Werks: Rekonstruktion

4. Schätzwert (bzw. Verkaufspreis) des Werks in der Quelle: Transkription bzw. Umrechnung (in Währungseinheiten)

B. Vertikale Anordnungen der Titel (in dreistufiger Reihenfolge)

Kriterium

1. Nutzen der Werke für den Besitzer, Interesse des Besitzers an den Werken
Anordnung

funktionell: (1) Orientierungslektüre, (2) Arbeitslektüre, (3) Bildungslektüre, (4) Unterhaltungslektüre, (5) Erbauungslektüre

\footnotetext{
23 Möglicherweise auch mit: Währungstabellen, Genealogien, Chronologien usw.

24 Vgl. N. Furrer, Des Burgers Buch..., S. 50, Tab. 10 (mit Anmerkungen).

25 Vgl. N. Furrer, Des Burgers Buch ..., S. 47, Tab. 7 (mit Anmerkungen).
} 
2. Zugehörigkeit der Werke zu einem Wissens- oder Fachbereich (Bildungslektüre), zu einer Thematik oder zu einer Textgattung

3. Verfasser bzw. Titel der Werke quantitativ: Von der grössten zur kleinsten Gruppe

alphabetisch: Von A bis Z

Tabelle 3: Funktionen der Lektüre in der frühen Neuzeit ${ }^{26}$
Lebensbereiche
Lektürearten
Funktionen
(1) Verwalten
Orientierungslektüre
Vergesellschaftung informative Lektüre
(2) Arbeiten
Arbeitslektüre
Lebenserhaltung, Einkom- professionelle Lektüre menssicherung
(3) Lernen
Bildungslektüre
Wissensvermehrung „szientifische” Lektüre
(4) Spielen
Unterhaltungslektüre
Lustgewinn, Entspannung
(5) Glauben belletristische Lektüre Erbauungslektüre
Gnadenerwerb, Trost religiöse Lektüre

Tabelle 4: Formale Buchbeschreibung in den Bibliotheksrekonstruktionen ${ }^{27}$

Elemente
Vorname(n)

Name(n)

Titel: Untertitel
Kennzeichnung Beispiel

Margarethe

Kapitälchen

kursiv
ZEERLEDER-LUTZ,

Glückselige Freyheit, entgegen gestellt der be-schwehrlichen

Dienstbarkeit, oder: Einfältige

Hertzens- und Erfahrungs-Lehr, einer durch

die Wahrheit frey gemachten

Schweitzerischen Frauen.

Für sie, ihre Kinder, und andere Heylsbegierige, hungrige Gnaden-Kinder. Zweyte Auflag.

Bern:

Gabriel Gaudard,

Verleger

(bei Berner Ausgaben)

26 Vgl. N. Furrer, Des Burgers Buch ..., S. 63, Tab. 17 (mit Anmerkungen).

27 Vgl. N. Furrer, Des Burgers Buch..., S. 48, Tab. 8 (mit Anmerkungen). 
Erscheinungsdatum

1743 ,

(Jahr oder Jahre)

Seitenzahl(en) bzw. An-

$256 \mathrm{~S}$

zahl Teile oder Bände

Format

in Klammern

(in-8).

Anmerkung: Erstausga- (Anmerkung)

Erstausgabe: Glückselige Frey-

be, weitere Ausgaben,

Originalausgabe

(bei Übersetzungen),

Hinweise auf biografi-

sche Informationen zum

Verfasser

heit, oder: Erfahrungslehre einer

schweizerischen Dame, die sich

durch Wahrheit frei gemacht. Neuwied, 1740. - Zur pietisitschen

Erbauungsschriftstel-lerin Marga-

rethe Zeerleder-Lutz (1674-1750)

siehe Guggisberg (1958) 412, 417, Bosshard (2008).

Tabelle 5: Die Dimensionen des geistigen Horizonts des frühneuzeitlichen Lesers $^{28}$

Dimensionen

räumliche Dimension

zeitliche Dimension

soziale Dimension

politische Dimension

kulturelle Dimension

Moral

(1) Sprache(n)

(2) Denkbereiche: Glauben, Ethik, Noetik, Ästhetik

(3) Wissens- oder Fachbereiche: Theologie, Recht, Medizin, Philosophie, Geschichte, Geografie, Naturwissenschaften

(4) Textgattungen: Traktate, Essays, Memoranden, Dialoge, Reden, Predigten, Reiseberichte, Briefe, Poesie, Theaterstücke, Romane, Hand- und Lehrbücher, Lexika und Wörterbücher, Zeitungen, Almanache, Mandate usw.

(5) Konfession, Religion oder Weltanschauung des Verfassers

historische Dimension Zukunftsträchtigkeit oder Dauerhaftigkeit der Werke

28 Vgl. N. Furrer, Des Burgers Buch ..., S. 66-67, Tab. 21 (mit Anmerkungen). 
Abbildung 1: N. Furrer, Des Burgers Buch: Stadtberner Privatbibliotheken im 18. Jahrhundert, Zürich 2012, S. 296.

2.14 Bibliothek des Berner Burgers und Almosenkammer- und Stubenschreibers

Wilhelm Rüetschi (1698-1743)29 anhand seines Geltstagsrodels vom 19. Dezember 1743

Nr. Titel im Inventar Beschreibung des Werks

Arbeitslektüre

1 Mutachs

verkürtzte Statt

Samuel Mutach, Substantzlicher Under-

Satzung

richt von Gerichts- und Rechts-Sachen, worinnen nach dem Methodo der Justinianischen Institutionen, so weit sich hat thun lassen der Statt Bern fürnehmste Justiz-Gesatz und Ordnungen eingebracht werden [...]. Denen so in dem Studio juridico einen Anfang machen wollen, zu etwelcher Anleitung und Erliech-terung zusammen getragen. Bern: [Selbstverlag], 1709, [10], 227, [22] S. (in-4). ${ }^{30}$

Bildungslektüre

(1) Geschichte, Politik

2 Memoires du Duc d'Ormont

[Thomas CARTE], Mémoires de la vie de Mylord duc d'Ormond, ci-devant capitaine-général \& commandant en chef des troupes de Grande-Bretagne. Traduit de 1'anglois. Den Haag, 1737, 2 Teile in $1 \mathrm{Bd}$. (in-8). ${ }^{31}$

3 Le Gouvernement civil

John Locke, Le gouvernement civil, oder: Die Kunst, wohl zu regieren. Durch den berühmten Engelländer Jean Lock beschrieben. Nunmehro aber [...] aus der Englischen und Frantzösischen Sprache in die Hochteutsche übersetzet von G. Frankfurt, Leipzig, 1718, [14], 513 S. (in-8). ${ }^{32}$ 
(2) Medizin

4 Recueil de Secrets [James De-La-Cour], Recueil de secrets et Remedes pour et remèdes tant pour guerir les maladies les maladies des du corps humain que pour celles des hommes et Bêtes bestiaux. Avec plusieurs remarques curieuses \& utiles

Abbildung 2: N. Furrer, Des Burgers Buch: Stadtberner Privatbibliotheken im 18. Jahrhundert, Zürich 2012, S. 514.

2.18. Der geistige Horizont des Berner «Quincaillerie Negotianten» Daniel Ludwig Hortin (1766-1806?) anhand seines Bibliotheksinventars vom 11. Juli 1798

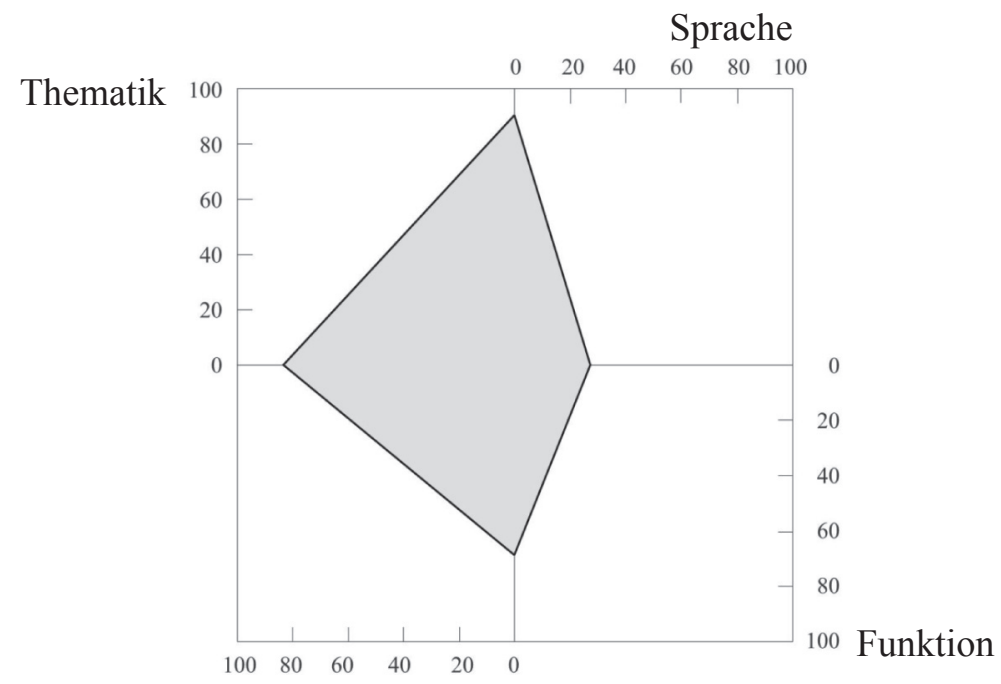

Alter

Thematik: Anteil der «weltlichen» (profanen) Werke an der Bibliothek: 91\% (105 von 115 Werken).

Funktion: Anteil der «extraprofessionellen» Werke an der Bibliothek: 68\% (78 von 115 Werken).

Alter: Anteil der «neuen» - in den letzten 50 Jahren vor dem Datum des Inventars verfassten bzw. erstmals publizierten - Werke an der Bibliothek: 83\% (96 von 115 Werken).

Sprache: Anteil der fremdsprachigen Werke an der Bibliothek: 27\% (31 von 115 Werken). 


\section{Zusammenfassung}

\section{Buchbesitz und geistiger Horizont. Zur Rekonstruktion frühneuzeitlicher Privatbibliotheken}

Will man den ,geistigen Horizonts” unserer Vorfahren historisch erforschen, kann man nach ihrem Buchbesitz fragen: Sag mir was Du liest, und ich sage Dir, wer Du - geistig - bist! Wie erfahren wir, welche Bücher bestimmte Menschen besassen? Insbesondere, wenn diese Menschen nicht jener schreibenden Elite angehörten, die verschiedenste Spuren ihres Lesens und anderer intellektueller Aktivitäten hinterlassen hat.

Zum persönlichen Buchbesitz in der Frühen Neuzeit finden sich oftmals Informationen in Nachlass- und Versteigerungsinventaren, von denen in verschiedenen Archiven der Schweiz längere Serien existieren, die um die Mitte des 17. oder im frühen 18. Jahrhundert einsetzen. Was man diesen Quellen entziehen kann, sind Listen von Buchtiteln. Will man diese Listen historisch analysieren, muss man aus ihnen - virtuell - Bibliotheken rekonstruieren.

Wie werden Bibliotheksrekonstruktionen gemacht? Welche Elemente soll die Rekonstruktion enthalten? Welches Maß an Vollständigkeit bei der Beschreibung der Werke ist erstrebenswert? Wie sollen die Werke angeordnet und wie die Bibliothek dargestellt werden?

Neben Forschungsstrategien braucht es auch Forschungstechniken, den Umgang mit einer Anzahl spezifischer Arbeitsinstrumente.

Bibliotheksrekonstruktionen ergeben untereinander vergleichbare Forschungsobjekte, auf die verschiedenste Analyseraster angelegt werden können. Erfolg versprechend scheinen insbesondere Raster zur Analyse des geistigen Horizonts der Buchbesitzer: sei es Menschen aller Art an einem bestimmten Ort, sei es eine bestimmte Kategorie von Menschen Pfarrherren, Kaufleute, Handwerker, Adlige, Bauern; Frauen - an verschiedenen Orten.

Schlüsselwörter: Schweiz im XVII-XVIII Jh - Privatbibliotheken - private Büchersammlungen - Geschichte des Lesens. 


\section{Streszczenie}

\section{Posiadanie książki i horyzont duchowy. Rekonstrukcja bibliotek prywatnych XVII-XVIII wieku}

Artykuł przedstawia propozycję metodologiczną wykorzystania inwentarzy spuścizn (ruchomości, licytacji) przy rekonstrukcji szwajcarskich bibliotek domowych z XVII-XVIII w. Autor zawarł w nim schemat postępowania badawczego opartego na nieznanych polskim księgoznawcom materiałach źródłowych pochodzących z archiwów kantonów Berno, Vaud, Jura, Neuchâtel i Solothurn. Zawierają one informacje na temat księgozbiorów prywatnych istniejących w badanym okresie zarówno w protestanckich, jak i katolickich, niemiecko- i francuskojęzycznych regionach Szwajcarii, w miastach i na wsi, których właściciele reprezentowali różne warstwy, grupy społeczne i zawody. Autor podkreśla, że rekonstrukcja dawnych bibliotek osobistych (lub prywatnych), ich typologia, liczebność, struktura tematyczna, funkcjonalna, językowa, a także aktualność (z punktu widzenia stosunku ilościowego dzieł starych do nowych) powinna prowadzić do określenia funkcji książki, lektury w życiu ich właścicieli. Umożliwić rozpoznanie ich horyzontów duchowych, zwłaszcza osób, które nie należały do ówczesnej elity umysłowej. Takie badania pozwalają również na uchwycenie tendencji rozwoju intelektualnego i kulturalnego społeczeństw w przeszłości.

Słowa kluczowe: Szwajcaria XVII-XVIII w. - biblioteki domowe - księgozbiory prywatne - historia czytelnictwa.

\section{Summary}

\section{Book possession and mindscape. The reconstruction of $17^{\text {th }}-18^{\text {th }}$ centuries personal libraries}

The article presents methodological proposition of using auction lists and inventories of an estate to the reconstruction of $17^{\text {th }}-18^{\text {th }}$ centuries' home libraries. Author proposes the scheme of methodological proceeding relied on source materials unknown to Polish book studies' scholars and stemmed from the archives of the cantons of Bern, Vaud, Jura, Neuchâtel and Solothurn Stoutt. These archives contain information on private book collections existing in discussed period, both in protestant and catholic re- 
gions of German- and French language parts of Switzerland, in the rural areas and in the cities, where the owners represented differentiated social milieus, social classes and occupations. Author stresses that reconstruction of personal (or private) libraries, their typology, numeral amount, their thematic, language, functional structure as well as topicality (seen as the balance between older books and newer ones) should lead to defining function of the book and reading in everyday life of libraries' owners. Such a libraries' reconstruction should also make possible diagnosing their owners' mindscape, specifically persons, who did not belong to intellectual elite of the times. These kind of research enables also to seizing the trends in intellectual and cultural development of the past societies.

Key words: Switzerland $17^{\text {th }}-18^{\text {th }}$ centuries - home libraries - private libraries - history of reading. 